FORMATION Formation emploi

Revue française de sciences sociales

146 | Avril-Juin 2019

L'apprentissage en Allemagne face à ses défis

\title{
Le/la formateur-rice : une position fragile. Étude de cas de grandes et moyennes entreprises, en Allemagne
}

The fragility of the trainer position - case studies in medium and large enterprises in Germany

Die Fragilität der Ausbilderposition - Fallstudien in mittleren und großen

Unternehmen

El formador : una posposición frágil. Estudio de caso de grandes y medianas

empresas en Alemania

\section{Anke Bahl}

\section{OpenEdition}

Journals

Édition électronique

URL : http://journals.openedition.org/formationemploi/7350

DOI : 10.4000/formationemploi.7350

ISSN : 2107-0946

Éditeur

La Documentation française

Édition imprimée

Date de publication : 26 juin 2019

Pagination : 53-75

ISSN : 0759-6340

Référence électronique

Anke Bahl, «Le/la formateur.rice : une position fragile. Étude de cas de grandes et moyennes entreprises, en Allemagne », Formation emploi [En ligne], 146 | Avril-Juin 2019, mis en ligne le 01 janvier 2021, consulté le 06 janvier 2021. URL : http://journals.openedition.org/formationemploi/7350 ; DOI : https://doi.org/10.4000/formationemploi.7350 


\title{
Le/la formateur.rice : une position fragile. Étude de cas de grandes et moyennes entreprises, en Allemagne
}

\begin{abstract}
ANKE BAHL Anthropologue sociale et chercheure en éducation et sciences sociales auprès de l'Institut fédéral pour la formation professionnelle en Allemagne (BIBB), au sein du département

"Développement des compétences»
\end{abstract}

Résumé

Le/la formateur.rice : une position fragile. Étude de cas de grandes et moyennes entreprises, en Allemagne

Au-delà des exigences formelles que les formateur-rice-s en entreprise du système d'apprentissage allemand doivent satisfaire, l'analyse menée dans cet article explore les motifs qui animent leur participation active au service de la formation au sein de l'entreprise. Des récits de plusieurs praticiens du monde de l'entreprise, il ressort une dynamique sociale complexe entre la direction, les formateur.rice.s et les apprenti.e.s. Le rapprochement des observations de la présente étude et de plusieurs études de cas françaises, menées dans et hors du système d'apprentissage, fait apparaître des similitudes étonnantes. Les conclusions révèlent que la formation sur le lieu de travail se conçoit comme une pratique d'ordre davantage social que pédagogique et soulignent la pertinence d'une culture du travail fondée sur la réciprocité et la confiance pour assurer la pérennité de l'apprentissage.

Mots clés: apprentissage, tutorat, culture professionnelle, enseignement techniqueprofessionnel, activité prof essionnelle, FPC en entreprise, étude de cas, Allemagne

Abstract

The fragility of the trainer position - case studies in medium and large enterprises in Germany

The paper looks behind the formal requirements in-company trainers in German apprenticeship have to fulfil and explores what keeps them actively involved in the training mandate of the firm. Based on accounts of various practitioners within the company context, a complex social dynamic between the management, the trainers and apprentices reveals. When contrasting the findings with a variety of French case studies both within and beyond apprenticeship schemes, surprising similarities appear. The results call for an understanding of workplace training as a social rather than educational practice and stress the relevance of a work culture based on reciprocity and trust for sustaining it. 
Keywords: apprenticeship, tutoring, professionalism, technical \& vocational education, vocational activity, CV'T in companies, case study, Germany

Journal of Economic Literature: M 51 ; M 53

Traduction : Auteure.

\section{Introduction}

La pérennité et la croissance d'une entreprise sur le plan économique sont, entre autres, fortement tributaires de sa capacité globale à attirer et à intégrer du personnel nouveau. La direction est certes responsable du recrutement et de l'emploi de ces nouveaux arrivants, mais c'est aux employé-e-s sélectionné-e-s qu'il revient de faciliter leur adaptation au nouvel environnement de travail et de les former dans l'entreprise (Barbier, 1996, p. 9 ; Nativel, 2016, p. 167). Le communiqué de Bruges (2010) qualifie les tuteur-rice.s et les formateur-rice.s de l'enseignement et de la formation professionnelles (EFP) d'acteurs-rice-s-clés de toutes les stratégies de l'Union européenne de promotion du développement socio-économique. Il appelle à une augmentation des investissements dans la formation d'ici 2020. Toutefois, jusqu'à présent, les avancées du secteur privé dans ce domaine sont faibles, ce qui soulève plusieurs questions dont se saisit la recherche.

Suite au regain mondial de l'apprentissage et à la popularité croissante des concepts d'orientation et de tutorat, de récentes études empiriques se sont intéressées aux pratiques réelles de formation sur le lieu de travail et ont proposé une analyse approfondie des dynamiques réciproques entre formateur.e.s et apprenti.e.s (Filliettaz, 2011 ; Kunégel, 2012 ; Boudet \& Triby, ainsi que Floro \& Raso et Nativel dans le dossier d'Education permanente, $\mathrm{n}^{\circ} 206,2016$, sur le tutorat). Bien qu'elles aient une influence décisive sur la nature et la qualité de la formation, les circonstances plus larges de cette pratique dans le cadre organisationnel particulier du monde du travail restent pourtant très peu étudiées.

Le présent article traite la fonction formatrice d'un point de vue structurel et analyse la position et la situation de travail des formateur.rice.s tout en tenant compte de l'entreprise, ainsi que du contexte juridique et économique dans sa globalité. En mobilisant des études de cas de formation en apprentissage en Allemagne, nous analysons la dimension subjective de la pratique de sélection des employés destinés à former les novices sur le lieu de travail. En quoi consiste le mandat de formateur-rice d'apprenti.e.s pour les formateur.rice-s sélectionné.e.s parmi le personnel ? Qu'est-ce qui nourrit leur motivation à former et la ravive dans le cadre de l'entreprise? 
Les résultats révèlent que les politiques allemande et européenne d'EFP ont pour inconvénient d'adopter une vision limitée des formateur-rice-s comme seuls agents et "pédagogues " individuels. L'analyse des récits des formateur-rice-s est structurée selon le modèle de "l'échange social tripartite ", esquissé à l'origine par une sociologue française (Maurines, 1997). Cette analyse, étayée par les résultats issus de plusieurs autres études de cas relevant de contextes différents, révèle la dynamique interne au mandat de formation qui est profondément imbriquée dans la culture du travail. Elle nous incite à concevoir la formation sur le lieu de travail comme une pratique d'ordre davantage social que pédagogique.

S’appuyant sur le cas allemand, cet article présente les caractéristiques propres au poste de formateur-rice dans le régime d'apprentissage de ce pays, ainsi que son cadre juridique (partie 1). Il présente ensuite la répartition générale des tâches de formation au sein du personnel, telle qu'elle est opérée dans la pratique, ainsi que les dilemmes qui en découlent (partie 2). Dans un premier temps, nous nous attachons à réunir les résultats épars de la recherche portant sur la configuration tutorale (3), avant de présenter le modèle industriel d'échange social (4). Les parties 5, 6 et 7 analysent la manière dont les rôles sont vécus par chacun des trois partenaires principaux de la mission de formation dans le cadre de l'entreprise, soit la direction, les formateur-rice-s et les apprenti.e.s. Nous confrontons alors l'idéal de pratique proposé, consistant en un échange mutuel de reconnaissance, aux exemples empiriques qui révèlent un changement de la culture du travail susceptible de menacer la position du/de la formateur-rice (partie 8). La conclusion synthétise les résultats pour ensuite inviter à revoir la politique d'EFP et à poursuivre les recherches.

\section{Le maître comme formateur : racines historiques et cadre légal dans le système moderne d'EFP}

La force du système allemand d'EFP réside notamment dans la tradition de formation sur le lieu de travail, ancrée de longue date dans la région et issue des guildes médiévales. Très tôt, les membres des corporations de métiers ont commencé à professionnaliser la transmission de leurs savoirs et leurs compétences propres afin de sécuriser et de pérenniser leurs activités, leur commerce et leur statut professionnel. Un apprenti devait consacrer plusieurs années au service de son maître pour recevoir l'autorisation de passer le certificat de compagnon et s'établir comme artisan à son compte. Par la suite, selon ses moyens financiers, la reconnaissance sociale et l'expérience professionnelle acquises au cours de plusieurs années supplémentaires, le compagnon pouvait obtenir le statut de maître et ainsi le droit d'engager lui-même des apprentis.

Si les structures d'organisation des guildes se sont lentement dégradées pour être finalement abolies sur le territoire allemand sous Napoléon au XIX ${ }^{\mathrm{e}}$ siècle, les pratiques 
de recrutement et de formation (pour les métiers d'art, d'artisanat et de commerce) ont perduré dans les entreprises de l'économie allemande. À l'aube du XX $\mathrm{XX}^{\mathrm{e}}$ siècle, les associations nationales de métiers se sont transformées pour s'adapter aux besoins des usines industrielles et du secteur des services, alors en plein essor, se dotant d'une composante publique ancrée dans le milieu scolaire et posant ainsi le fondement de l'EFP en alternance.

L'État-nation étant devenu un acteur de l'enseignement professionnel, et à la faveur du développement des écoles professionnelles, la fonction de « professeur d'EFP » a vu le jour. Toutefois, la formation de la relève reste majoritairement du ressort du secteur privé et seuls les maîtres sont autorisés à dispenser la formation. Cette tradition s'incarne encore dans la législation du système moderne d'EFP allemand (cf. Le Mouillour \& Geiben 2016). Afin d'assurer la continuité de la formation en entreprise, la Loi sur la formation professionnelle stipule que toute entreprise offrant de la formation à un apprenti sous contrat doit disposer d'au moins un employé nommé formateur-rice référent.e (le/la tuteur.rice ou maitre d'apprentissage, en allemand "Ausbilder»), dont l'aptitude à assumer cette fonction est attestée. Pour attester de cette qualification, outre la qualification professionnelle, il faut réussir un examen, conformément au règlement relatif à l'aptitude de formateur ("Ausbilder-Eignungsverordnung" (AEVO)). Les formateurs aux métiers artisanaux qualifiés sont généralement des maîtres artisans car, dans la lignée de la tradition historique des guildes médiévales, l'examen pour prétendre au diplôme de maître artisan comprend généralement un test d'aptitude de maître d'apprentissage. Pour les métiers non artisanaux, tout formateur·rice qualifié.e conformément à l'AEVO est accepté.e (cf. Hensen \& Hippach-Schneider 2016).

\section{La formation : une mission difficile, à la croisée de l'enseignement et du monde du travail}

Quelles sont les implications de ce cadre juridique pour les formateur·rice-s désigné.e.s comme référent.e.s ? Ils/elles sont tenu.e.s, d'une part, de remplir une mission de service public encadrée par le règlement du système d'EFP. Il leur faut préparer les jeunes afin qu'ils obtiennent le diplôme de formation professionnelle et réussissent l'examen de la chambre du métier choisi. Ils/elles sont tenus de respecter les règlements standard dictés par l'État, soit la Loi sur la formation professionnelle (BBiG $\$ 14)$. Ils/elles doivent, d'autre part, développer une nouvelle main-d'œuvre composée de travailleurs bien qualifiés et loyaux, répondant aux besoins de l'entreprise. Pour atteindre ce double objectif, ils/elles sont tributaires de la motivation de chacun.e de leurs apprenti.e.s. Pour ce faire, répondre aux besoins personnels des apprenti.e.s et trouver le bon équilibre entre les objectifs économiques et pédagogiques sont des conditions nécessaires. 
Les tuteur.rice·s ou maîtres d'apprentissage se situent à la croisée des systèmes d'enseignement et d'emploi et sont contraints d'équilibrer les attentes conflictuelles vis-à-vis de ce double rôle et des responsabilités qu'ils assument. À l'inverse des enseignants des écoles d'EFP, leurs profils de poste ne sont généralement pas pédagogiques, mais bien professionnels et liés à leur fonction dans les activités de l'entreprise. En outre, la formation constitue une activité secondaire pour la majorité des tuteur·rice·s, puisque ceux qui exercent à plein temps et qui se consacrent principalement à la formation sont très minoritaires. La sécurité de leur emploi dépend avant tout de la réussite économique de l'entreprise.

Pour les apprenti.e.s, la formation dans le cadre de l'apprentissage implique de se familiariser avec tous les processus de travail pertinents pour leur métier. À cet égard, le/ la tuteur-rice référent.e est tributaire de l'éventuelle propension des autres employé.e.s de l'entreprise à transmettre leurs connaissances et leurs compétences spécifiques, tandis que les apprenti.e.s tournent dans les différents départements de l'entreprise. Il existe donc de grandes disparités quant aux types de tuteur.rice-s et à leurs profils selon l'entreprise allemande qui propose une formation en apprentissage. La singularité de chaque métier, ainsi que l'affiliation sectorielle de l'entreprise et les postes et lieux de travail existants influent sur la structure de la formation. Plus l'entreprise est grande, plus la répartition interne des tâches est complexe.

Dans les petites entreprises de 15 à 25 employés, la formation présente en pratique de nombreux points communs avec la formation autrefois dispensée chez le maître (Bahl, 2018). Dans la plupart des cas, le/la chef.fe d'entreprise assure le rôle du/de la tuteur-rice référent·e, mais n'a pas à lui/elle seul.e le temps de former et d'observer le novice en permanence. Il/elle délègue donc des tâches de formation à ses compagnons et autres collègues maîtres artisans et c'est toute l'équipe qui unit ses forces pour s'occuper de l'apprenti-e. Dans les petites entreprises, tous les employé.e.s participent plus ou moins au processus de formation, tandis que le/la chef.fe d'entreprise est formellement responsable et supervise la pratique de formation.

Comment la formation est-elle pratiquée dans les entreprises moyennes et grandes ? Selon le nombre d'apprenti.e.s en formation, la direction doit nommer un ou plusieurs employé.e.s chargé.e.s d'être le.s formateur-rice.s officiel.le.s ou tuteur.rice.s référent·e.s. La mission de formation est déléguée à des employé.e.s choisi.e.s. Le/la tuteur.rice référent.e est formellement responsable, mais ici aussi, il/elle est en réalité tributaire du soutien collectif des personnels des autres départements de l'entreprise.

En outre, le/la formateur.rice est responsable du contrôle des apprenti.e.s, considéré.e.s comme de nouveaux employé.e.s de l'entreprise, ce qui le/la place dans une position inconfortable au sein de la hiérarchie de l'entreprise. Il/elle demeure, d'une part, un·e employé·e dépendan·e tout en devenant, d'autre part, le/la supérieur·e de l'apprenti.e. Du fait de leur mission particulière et de leur position intermédiaire mi-employeur 
mi-employé, les formateur·rice.s d'apprenti-e.s sont souvent peu soutenu.e.s institutionnellement par le conseil d'entreprise ${ }^{1}$. L'ambivalence et la précarité de leur statut (cf. Schlösser, Drewes \& Osthues, 1989) d'agent pédagogique au sein de l'entreprise ont conduit la recherche allemande sur l'enseignement à qualifier "d'institutionnellement faible " la position de tuteur.rice référent.e ou maître-sse d'apprentissage en entreprise (Pätzold, Drees \& Lietz, 1986). Ce phénomène apparaît dès lors que la responsabilité de la formation est déléguée à des collègues ou des départements particuliers de l'entreprise et cesse d'être portée collectivement. En conséquence, il prévaut dans les entreprises moyennes et grandes, tous secteurs confondus.

Ainsi, les tuteur·rice.s référent.e.s dans ces entreprises assument une mission particulièrement difficile. Ils doivent non seulement remplir des fonctions pédagogiques dans le contexte du monde du travail où les motivations sont avant tout économiques, mais sont en outre tributaires de la collaboration et du soutien de nombreux autres collègues et partenaires au sein de l'entreprise et en dehors de celle-ci.

La partie suivante s'appuie sur des études de cas représentatifs, menées dans diverses entreprises allemandes pour analyser les implications des caractéristiques de la mission de formation sur les personnes concernées. Comment les tuteur·rice-s vivent-ils/ elles leur rôle et comment le gèrent-ils/elles ? Qu'est-ce qui stimule et nourrit leur motivation à former dans le cadre de l'entreprise ? Quelles approches théoriques correspondent aux schémas révélés par l'analyse par reconstitution des récits des personnes interrogées ? Quelles sont les analogies entre les résultats de cette analyse et les recherches sur la formation sur le lieu de travail dans le cadre d'autres entreprises, en France et ailleurs?

1. La Loi allemande sur la formation professionnelle (BBiG $\$ 27-30)$ et la loi sur les comités d'entreprise (BetrVG $\$ 98$ ) protègent les apprenti.e.s des employeurs et des tuteur-rice-s ne possédant pas les aptitudes personnelles requises. Toutefois, les membres des conseils ou comités d'entreprise ont traditionnellement tendance à prendre parti pour les apprenti.e.s en cas de conflit et sont peu ou mal informés des contraintes que comporte la fonction de tuteur.rice (cf. Busse, 2011). 


\section{Encadré 1. Méthodologie}

Les données qualitatives mobilisées pour le présent article sont issues d'un projet de recherche national intitulé « La situation des formateurs d'apprentis en entreprise ». Cette étude a été menée entre 2009 et 2012, pour le compte de l'Institut fédéral pour la formation professionnelle (BIBB) et comprend quatorze études de cas d'entreprises réparties dans toute l'Allemagne (Bahl \& Blötz, 2012). Elle vise à mieux connaître les obstacles au développement professionnel et au soutien des effectifs de formation participant à l'apprentissage dans le secteur privé.

Les entreprises formatrices étudiées présentent de fortes disparités en termes de taille, de secteur, d'années d'expérience dans la formation en apprentissage, de région et de soutiens complémentaires provenant de prestataires de formations externes. Suivant l'approche de l'échantillonnage pour la " théorie ancrée » (grounded theory), les études de cas ont été choisies et effectuées l'une après l'autre en trois vagues. Dix à quinze entretiens ont été menés dans huit entreprises, pour un à six entretiens dans les autres entreprises. Trois grandes entreprises de plus de 1500 employés ont été investiguées, cinq entreprises de taille moyenne (entre 500 et 1000 employés) et six petites entreprises (de six à 90 employés). Elles relèvent des secteurs d'activité commerciale et industrielle suivants : énergie et ingénierie électrique, construction automobile et construction de moteurs, industrie chimique, fabrication d'outils, systèmes et composantes de conduite, gestion d'équipements (nettoyage), construction, assurances, technologies de l'information, hôtellerie et restauration, santé dentaire, artisan électricien, artisan véhicules à moteur.

L'équipe a mené au total 127 entretiens semi-directifs auprès d'employé.e.s des catégories suivantes:

- Chefs d'entreprise / directrices et directeurs des ressources humaine/ directrices et directeurs des départements de formation :

- Formatrices et formateurs à plein temps et à temps partiel ;

- Supérieurs hiérarchiques;

- Déléguéee.s du personnel ;

- Apprenti.e.s .

Les entretiens ont duré en moyenne une heure chacun. Le logiciel MAXQDA a été utilisé pour les fichiers audio et les transcriptions. Les récits ont fait l'objet d'une interprétation herméneutique afin de reconstituer les expériences particulières des personnes interrogées et d'élaborer une première esquisse de « théorie [empiriquement] ancrée » de la situation des formateurs sur le lieu de travail, au sein du système allemand d'apprentissage.

À l'issue du projet, l'auteure a continué d'utiliser les données pour des recherches ultérieures. Sa thèse de doctorat (Bahl, 2018) consiste en une analyse et un compte rendu ethnographique de la pratique de formation dans différents métiers d'après la description des formateurs. 


\section{Obligations ressenties par les formateur.rice.s au sein d'un réseau complexe : « la configuration tutorale »}

Les études de cas ont pour principal objectif (voir encadré 1 pour la méthodologie) de décrire la pratique réelle des formateurs dans le monde du travail. Les schémas qui se sont dégagés de l'écoute répétée des convictions personnelles et des explications des formateurs, de leurs supérieurs et des apprenti.e.s ont révélé une dynamique sociale primaire de la formation. Une citation-clé illustre le travail de reconstitution et d'herméneutique effectué à partir des récits des personnes interrogées. Lorsqu'il est interrogé sur son rôle dans le cadre de l'entreprise, le directeur de formation d'une grande entreprise du secteur automobile, très respecté et récemment parti à la retraite, recourt ainsi à une métaphore :

"Vous êtes toujours pris entre le marteau et l'enclume. Vous avez des obligations vis-à-vis de la direction, bien sûr. Vous avez également des obligations vis-à-vis de l'apprenti, du comité d'entreprise et des parents. Vous devez rester très stable et garder les idées claires lorsque vous abordez les choses. (...) Ceci requiert des compétences personnelles considérables (...), à la manière d'un homme politique, (...) pour faire en sorte que tout le monde soit content dans une certaine mesure. " [10_01_HA, 426] ${ }^{2}$

Cette description évoque non seulement la position particulière d'intermédiaire dans laquelle se trouve le tuteur.rice d'apprenti.e.s, mais fait également allusion à l'importante dimension sociopolitique qu'elle comporte. Manifestement, la description de poste formelle ne suffit pas à saisir avec exactitude cette fonction, pas plus qu'elle ne permet de l'exécuter techniquement en conséquence. En effet, afin d'être reconnue et menée à bien, cette tâche requiert une négociation constante et de la diplomatie avec de nombreux partenaires au sein de l'entreprise et en dehors de celle-ci. Bien qu'elle soit responsable uniquement devant la direction et tenue de lui rendre des comptes, la personne interrogée cite plusieurs autres parties prenantes dans sa fonction de formateur.rice, dont les apprenti.e.s et leurs parents. Si elle ne mentionne pas explicitement toutes les attentes qui pèsent sur elle, un certain nombre d'obligations, y compris de nature morale, ressortent de sa description.

À première vue, le statut des tuteur.rice-s allemands d'apprenti.e.s semble évident : ils/ elles doivent être formellement inscrit.e.s en tant que tel-le.s auprès des chambres de commerce et d'industrie ou des chambres des métiers et leurs fonctions sont prescrites par la législation de l'EFP. L'entreprise les recrute au sein du personnel pour cette tâche et ceci est généralement enregistré dans le dossier personnel. Il existe donc un accord formel. Cependant, dans la pratique quotidienne, la formation en apprentissage suit une logique qui va bien au-delà. Il n’y a pas de simple délégation de tâches du sommet

2. Ce code fait référence à la retranscription de l'interview (code anonyme pour l'entreprise et la personne interviewée) et indique la ligne précise (426) dans le corpus de MAXQDA. 
de la hiérarchie au formateur-rice, ni de simple transmission de savoirs et de compétences de l'expert au novice. Le cœur du processus de formation semble caractérisé par des obligations et une confiance mutuelles, davantage que par un lien essentiellement unilatéral et économique.

D'autres études portant sur les pratiques d'apprentissage, en Allemagne et ailleurs, soulignent également la pertinence d'un grand nombre de partenaires dans le cadre de cette activité. Pelé-Peycelon \& Veillard (2016) ont mené une observation systématique des interactions de deux formateur-rice-s avec leur supérieur hiérarchique, l'apprenti-e et le/la tuteur-rice de l'école d'EFP. Les auteurs sont partis d'études de cas, en France, pour identifier quatre principaux partenaires liés au poste de formateur-rice : l'apprenti.e, les collègues, la hiérarchie et le/la tuteur-rice-école. Selon la notion de "configuration" de Norbert Elias, définie comme un "réseau d'interactions et d'interdépendances", ils ont proposé le terme de "configuration tutorale " pour cerner la position du/de la formateur.rice :

"Nous considérons que le MAP [Maître d'apprentissage] est engagé dans une 'configuration tutorale' qui renvoie aux interdépendances développées avec différents acteurs, internes ou externes à l'entreprise, pour assurer le tutorat de l'apprenti. (...) La configuration peut être dépendante de l'organisation du travail dans l'entreprise, des pratiques tutorales déjà existantes, mais aussi d'éléments extérieurs apportés par l'organisation de la formation par apprentissage. " (Pelé-Peycelon \& Veillard, Ibid., p. 71 s.)

La notion de configuration est effectivement utile pour saisir la diversité des attentes ${ }^{3}$, parfois même contradictoires, et des partenaires auxquels le formateur.rice est confronté. Toutefois, elle n'explique pas l'aspect central de cette activité exercée habituellement à titre volontaire. Quelle est la dynamique inhérente à cette " configuration tutorale "? Qu'est-ce qui fait son caractère unique au sein de l'environnement de travail ? Qu'est-ce qui stimule la motivation à former et l'alimente dans le cadre de l'entreprise ? Enfin, quels modèles théoriques existants apportent des réponses à ces questions ? La notion "d'échange social», mise en avant par Peter M. Blau (1964) pour comprendre la structure sociale, s'avère très utile à cet égard, comme nous le verrons dans le développement qui suit. Des études de cas datant des années 1990, menées dans l'industrie chimique française, ont permis à la sociologue Béatrice Maurines d'affiner cette notion et de l'étendre à la pratique de formation sur le lieu de travail.

3. Pour les tuteur.rice-s suisses d'apprenti.e.s, Besozzi, Perrenoud \& Lamamra évoquent « la double casquette» et «la nécessité de penser et d'articuler différents registres d'activités" (2017, p. 59). 


\section{Le modèle des trois partenaires de l'échange social, de Béatrice Maurines}

Béatrice Maurines a mené des observations très intéressantes dans les usines industrielles françaises des années 1990 (Maurines, op. cit.). ${ }^{4}$ Alors qu'elle étudiait l'introduction de nouveaux ouvriers, elle s'est rendu compte que la transmission de savoirs et de compétences des experts aux novices était fortement tributaire d'une relation d'échange social et de confiance entre les deux parties. Ce résultat a été confirmé par de nombreuses autres études consacrées aux relations de formation (Barbier, op. cit., p. 13 ; Nägele \& Neuenschwander, 2016 ; Kunégel évoque même un "phénomène d'adoption réciproque" pour la formation en apprentissage, op. cit., p. 10).

Maurines étend toutefois son analyse au-delà de la dynamique immédiate entre expert et novice, pour envisager la position du/de la formateur-rice vis-à-vis de la totalité des travailleurs de l'entreprise. Reprenant la notion bilatérale d'échange social mise en avant par Blau ${ }^{5}$, en l'envisageant dans un cadre économique à forte dimension hiérarchique, elle suggère d'inclure un troisième partenaire à ce modèle d'échange mutuel, soit la hiérarchie : "La transmission (...) s'appuie sur différents protagonistes aux statuts différents : la personne en formation, le formateur, l'encadrement hiérarchique." (Maurines, op. cit., p. 9). Elle évoque une relation tripartite dans le contexte industriel et développe un modèle comprenant trois partenaires de l'échange social qui explique le caractère étonnamment volontaire de la pratique de formation : "L'obligation de formation des partenaires n'est spécifiée ni dans le contrat de travail, ni par écrit, ni même sur le cahier des équipes rédigé quotidiennement par la hiérarchie où apparaissent les activités à exercer. Il s'agit donc d'une relation interpersonnelle, même si les partenaires ne sont pas statutairement égaux dans l'entreprise. " (Ibid.)

Malgré les différences évidentes entre le système formel d'apprentissage et l'assimilation de compétences dans la formation de nouveaux ouvriers, et bien que le système allemand d'apprentissage comporte une obligation de se conformer à la législation de la formation, notre analyse révèle que la réussite de la formation dépend fortement

4. Elle a mené un travail de terrain pendant cinq ans, dans plusieurs entreprises du secteur chimique de la région Rhône-Alpes au Sud de Lyon et étudié le travail et les pratiques de formation dans huit unités de production différentes.

5. Comme l'a souligné Blau, "des obligations non spécifiées distinguent l'échange social de l'échange strictement économique ". Tandis que l'échange économique est spécifié et fondé sur un accord formel afin de garantir que chacune des parties remplisse ses obligations spécifiques, l'échange social comprend des obligations non spécifiées : "L'échange social en revanche repose sur le principe qu'une personne rend service à une autre et sill existe une attente générale de retour à venir, la nature globale de ce retour n'est absolument pas établie à l'avance. " (1964, p. 93). Ceci fait écho à la motivation que de nombreux formateur.rice.s tirent de leur propre parentalité. Ils souscrivent à leur obligation de former, car ils souhaitent que leurs propres enfants soient bien formés eux aussi. 
des relations informelles et de la collégialité entre les membres du personnel. Comme mentionné ci-dessus, un·e formateur·rice ne peut à lui/elle seul·e remplir la mission de formation ; cela dépend du soutien apporté volontairement par de nombreux autres collègues dans différents départements de l'entreprise. En outre, pour maintenir sa motivation, le/la tuteur-rice dépend également du soutien des partenaires, notamment de la direction. Le caractère interpersonnel de la relation entre plusieurs partenaires impliqués dans la mission de formation mérite donc que l'on s'y attarde.

Kunégel propose la répartition des rôles suivante pour la formation des apprenti.e.s sur le lieu de travail, en France : "Le tuteur hiérarchique (l'employeur) embauche et rémunère l'apprenti. Il est l'interface entre la famille, le CFA et l'atelier. Le tuteur relais (le chef d'atelier) organise le travail. Il peut confier des travaux à l'apprenti ou le confier au tuteur opérationnel qui est directement en contact avec le jeune. " (op. cit., p. 2). Il serait tentant d'attribuer la fonction de tuteur à tous les partenaires impliqués dans la mission de formation. Ceci pourrait pourtant masquer le contexte économique motivé par la rentabilité de l'organisation et soulève aussi des difficultés dès lors que l'on souhaite saisir chacune des relations en tant que telle.

Maurines a identifié trois partenaires centraux de l'échange social. Bien que la « configuration tutorale » de l'apprentissage soit plus complexe que cela, comme nous l'avons vu, et qu'elle implique également, dans le cas allemand, d'autres partenaires tels que les chambres des métiers, le comité d'entreprise (le cas échéant), les enseignants de l'école d'EFP et les parents, le triangle formé par 1) la direction, 2) le/la tuteur-rice et 3) l'apprenti.e comprend les principaux partenaires au sein de l'entreprise. Nous allons à présent adapter le modèle de configuration tutorale de Maurines au cas de l'apprentissage en Allemagne et sonder sa valeur afin de mieux analyser la dynamique interne du mandat de formation. Pour commencer, nous aborderons le point de vue de la hiérarchie, c'est-à-dire la direction de l'entreprise. 


\section{Graphique 1. Interdépendance de la position de formateur.rice selon le modèle tripartite d'échange social}

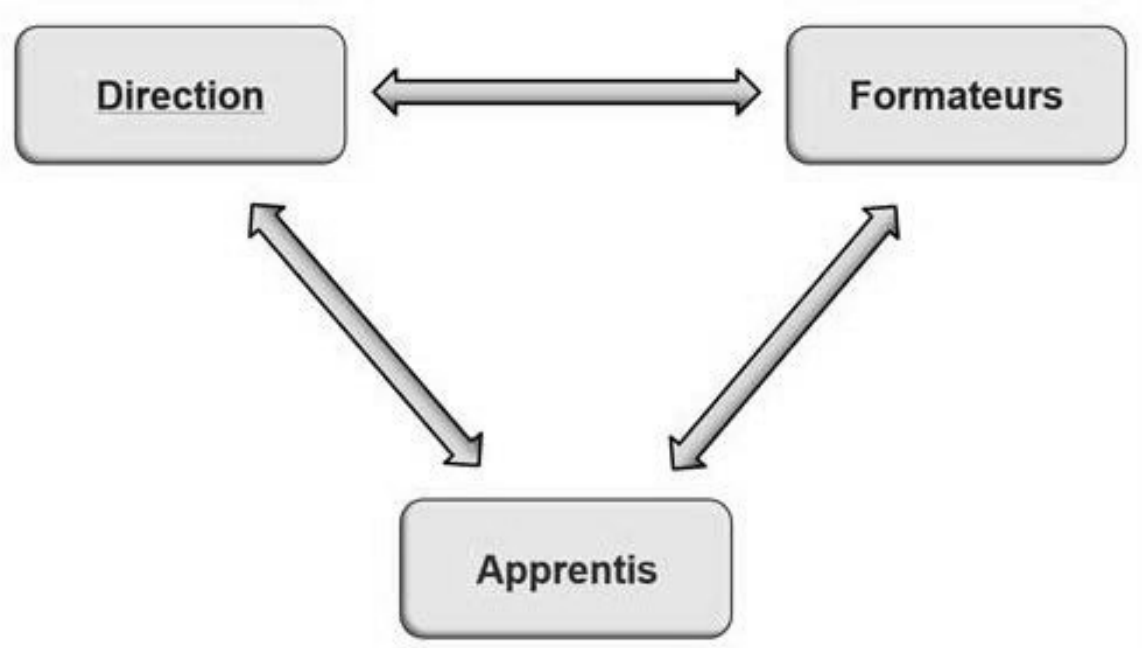

Source : Auteure.

\section{Critères de sélection des formateur.rice.s selon la direction}

Nous présentons ici le résultat de l'analyse qualitative. D’abord, les résultats généraux pour une pratique de formation qui fonctionne (parties 5 à 7) ; ensuite, les menaces qui pèsent sur cette pratique et qui ont été identifiées dans des cas précis (partie 8) ; enfin, les conclusions générales sur le soutien à la position de formateur-rice.

L'essentiel de la logique de la direction peut être décrit comme suit : pour pérenniser leurs effectifs et leur compétitivité, les entreprises (notamment les grandes et les moyennes) dépendent des employé.e.s-formateur.rice.s, qui sont qualifié.e.s pour ce faire et n'exigent pas trop de contrepartie pour réaliser cette tâche supplémentaire. Du point de vue industriel et économique, certains critères de sélection de ces employé.e.s s'avèrent plus pertinents et ont donc été cités à plusieurs reprises par les représentants de la direction interrogés.

La possession d'un certificat d'aptitude de formateur.rice (AEVO) s'est étonnamment avérée moins pertinente que ne le laissent entendre les déclarations officielles (Bahl \& Brünner, 2013, p. 523 s.). Pour être conforme aux règles du système d'EFP, la direction reconnaît en général formellement cette exigence. Toutefois, les praticiens ont une notion différente de « l'aptitude », davantage en lien avec des caractéristiques sociales. 
Quiconque qui aspire à former une main-d'œuvre de relève qualifiée devrait, en toute logique, rechercher des personnels à la fois dotés d'une solide connaissance du métier visé et fidèles à l'entreprise, car ils feront figure d'exemple pour les novices. Toutefois, en pratique, il s'avère que les personnels dotés de qualifications formelles portant sur le métier et la pédagogie ne sont pas tous volontaires pour partager leur savoir avec d'autres. ${ }^{6}$ Certaines personnes ont déclaré se sentir menacées par les nouveaux arrivants, craignant d'être licenciées si elles investissaient dans un collègue (souvent plus jeune). D'autres préferent en général travailler seules et sont peu enclines à expliquer à autrui ce qu'elles font et à dispenser de la formation. Ce propos d'un formateur.rice à plein temps d'une entreprise de métallurgie résume bien la situation :

"La plupart des départements considèrent que l'apprentissage est très important. Ils apprécient de recevoir des personnes bien formées, mais en réalité, la plupart des départements ne souhaitent pas prendre en charge eux-mêmes la formation.» [08_06_HA, 250]

C'est pourquoi il est essentiel que la direction recrute des personnes qui sont déjà enclines à assurer cette mission, qui manifestent de l'engagement vis-à-vis des jeunes et, dans le meilleur des cas, qui n'attendent pas de rémunération financière, mais se portent volontaires pour assumer ces tâches supplémentaires.

Dans les grandes et moyennes entreprises allemandes, en raison de la grande variété des profils de formateur.rice.s, la direction les recrute généralement en concertation avec la personne désignée responsable de la formation. Dès lors, la frontière entre la direction et les formateur.rice.s s'estompe.

La majorité des formateur·rice-s interviewé·e.s ne s'étaient pas porté·e.s candidat·e.s dès le départ pour assumer cette fonction, ce qui est typique de l'apprentissage en Allemagne (cf. Bahl, 2012, p. 29 s.), mais ont été sollicité.e.s par leurs supérieurs qui leur ont demandé s'ils/elles envisageaient d'assumer ce rôle. La formulation fréquemment employée est "Cela s'est fait comme ça", laissant entendre que le passage au poste de formateur-rice est une sorte de " progression naturelle».

Le cas suivant illustre parfaitement la pratique habituelle et donc « naturelle » qui sous-tend ce processus. Questionnée sur la méthode employée pour identifier et recruter les employés des unités de production qui se prêteraient à la formation, une responsable de formation chevronnée, travaillant dans le secteur de la chimie en Allemagne de l'Est répond :

"Dans certains cas, on connaît déjà les collègues des usines, du moins les plus âgés, on reçoit également les retours des apprentis. Lorsque l'on entend des apprentis dire des choses comme: 'Oui, cet homme a été mon superviseur, c'était fantastique, il m'a montré tant de choses', alors oui, je me dis 'C'est intéressant, on ne connaît pas encore ses qualités de formateur'. Ensuite, je le contacte pour commencer; si c'est un nouveau collègue, je le contacte et nous avons un entre-

6. Pour des résultats similaires dans des études de cas consacrées à la formation sur le lieu de travail en Australie et au Royaume-Uni, cf. Billett, 2002, p. 463. 
tien individuel. Ensuite, je vais voir le directeur de l'usine et je lui dis 'C'est un bon tuteur, pourquoi ne pas lui faire faire davantage de supervision ?" [07_07a_HA, 400-402]

Cette directrice de formation utilise manifestement ses échanges informels avec les apprenti-e.s pour se faire une idée du quotidien du travail dans les différents départements. Lorsqu'ils/elles relatent des expériences positives de collègues qui les ont aidé.e.s et les ont guidé.e.s d'une manière ou d'une autre, elle demande leur nom. Si elle ne les connaît pas encore personnellement, elle les invite à prendre le café dans son bureau afin d'en savoir davantage sur leur attitude vis-à-vis du travail, de la formation et des jeunes. Si elle a une bonne impression, elle prend contact avec le/la supérieur·e hiérarchique de l'équipe pour lui demander ce qu'il/elle en pense. Si les deux s'accordent, la personne en question recevra une demande formelle pour officier comme formateur.rice.

Cette pratique de recrutement correspond parfaitement à l'analyse des dynamiques menée par Maurines dans les usines chimiques en France : "Le formateur est sollicité par la hiérarchie; cette dernière a dî̀ évaluer à l'avance ses capacités à la formation, mais aussi détecter si elle pouvait avoir confiance en ce partenaire." (op. cit., p. 9). Toutefois, les autres études effectuées en France et citées dans le présent article n'abordent pas explicitement la pratique de recrutement en tant que telle. ${ }^{7}$

\section{La formation comme gain d'autonomie, de confiance et d'estime de soi}

Que retire le formateur-rice de sa nouvelle fonction? Dans l'idéal, un des avantages de ce rôle est la reconnaissance que vous accorde la direction, qui voit en vous un employé compétent et de confiance. Pour la majorité des formateur·rice.s interrogé.e.s, la formation constitue une activité secondaire. Ils/elles sont nombreux·ses à apprécier cette tâche supplémentaire, car cela les change de leur routine professionnelle habituelle.

En outre, le rôle de formateur.rice leur apporte une certaine autonomie tant dans la définition que dans la réalisation de cette nouvelle tâche. Ce résultat est surprenant dans la mesure où le système allemand d'EFP prévoit des règlements de formation détaillés pour tous les métiers. Pour chaque métier, le règlement comporte un programme général et un calendrier de formation et la plupart des entreprises les adaptent à leurs propres processus opérationnels.

\footnotetext{
7. Floro \& Raso (op. cit.) écrivent tout au moins que « la question du recrutement des tuteurs et de leur formation est un incontournable qui exige des approfondissements ultérieurs" (p. 94). Nativel (op. cit.) mentionne que "le rôle du tuteur est plus au moins imposé aux managers de proximité (agents de maîtrise) qui, compte tenu de leur expérience, sont censés disposer des qualités requises pour gérer leurs équipes " (p. 167).
} 
Nos études de cas ont toutefois révélé que la majorité des formateur·rice·s ne travaillent pas avec ces programmes et ont davantage tendance à s'appuyer sur leurs propres convictions et leur propre fierté professionnelle pour décider de ce qu'il convient de transmettre ou d'enseigner dans leur département. Un agent administratif d'un grand groupe d'assurances allemand résume bien cette attitude :

"Non, la direction de mon département [c'est-à-dire le supérieur hierarchique direct] me laisse largement le champ libre, il me fait confiance et les retours positifs des apprentis le convainquent que les choses sont gérées correctement. Les résultats aux examens sont bons, cela signifie qu'il n'y a pas de raison de me donner davantage de prescriptions ou des consignes différentes. [...] Moi aussi, je trouve que c'est une bonne chose qu'il me laisse relativement le champ libre et qu'il n'influence pas mon travail ou la manière dont je l'exerce." [02_06_NA, $135,201]$

Ce formateur-rice apprécie l'autonomie que lui confère sa position. La directrice de formation de l'ensemble du groupe d'assurance, répondant à nos questions sur les consignes ou les directives relatives à la pratique des formateur.rice.s, explique :

"En fait, tout le processus de sélection [des apprentis] est pré-stipulé. Ces questions sont fixées par écrit. Par contre, ce qui se passe dans l'intermédiaire, la formation au quotidien, dans le département, dans les [bureaux de vente], au sein de l'unité, requiert une prise de décision individuelle et imaginative. À cet égard, nous ne pouvons pas influencer le processus. Et nous ne cherchons pas à le faire. Nous préférons aborder le sujet de manière convaincante. " [02_15_HA_FK, 521]

Cette déclaration illustre bien à quel point la direction, dans ce cas la directrice de formation, est tributaire de la motivation individuelle et continue des formateur.rice.s qu'elle a nommés. Il est suffisamment difficile de recruter des personnels qui se portent volontaires pour assumer cette mission supplémentaire, les consignes qui leur sont imposées sont donc réduites autant que possible.

Une fois de plus, malgré les différences de cadre légal entre l'Allemagne et la France, nous parvenons aux mêmes conclusions que Maurines. Les personnels sont chargés euxmêmes d'assurer la reproduction de la main-d'œuvre. Maurines écrit (op. cit., p. 10) :

"La hiérarchie, tout d'abord, fait confiance au formateur pour ses capacités de pédagogue, au formé pour sa capacité d'attention et sa volonté d'apprendre son nouveau travail. La hiérarchie se décharge en quelque sorte de cette responsabilité; elle laisse le collectif de travail gérer cette formation informelle, à l'exception du schéma de procédé." 


\section{L'interdépendance entre formateur.rice et apprenti.e}

Qu'en est-il de la dynamique entre formateur·rice et apprenti·e ? Les formateur.rice.s deviennent une figure d'autorité importante pour les apprenti.e.s. Ils/elles disposent de connaissances et de compétences essentielles aux qualifications que les novices souhaitent acquérir. Les novices dépendent à leur tour du soutien et des conseils des experts de la profession, tandis qu'ils se familiarisent peu à peu avec ce nouveau monde du travail.

Le degré de soutien que reçoivent les apprenti-e.s dépend des contextes de leur lieu de travail, de la personnalité du/de la formateur.rice et des cultures d'apprentissages des uns et des autres. Toutefois, l'attitude de l'apprenti.e a elle aussi un impact. Plus il/elle affiche intérêt et curiosité pour l'apprentissage, plus il/elle s'implique afin de réaliser le travail de manière autonome (cf. Duc, Perrenoud \& Lamamra, 2018, p. 7s.), plus le/ la formateur-rice sera généralement disposé.e à lui transmettre ses connaissances et à lui donner accès à d'autres possibilités d'apprentissage et d'autres tâches intéressantes.

La dynamique mise à jour par notre étude, en Allemagne, correspond en tout point à la déclaration de Kunégel : "Un apprenti sera d'autant mieux suivi, conseillé, accompagné qu'il aura réussi à se faire accepter par le tuteur et les autres membres du collectif de travail. » (op. cit., p. 109)

Le statut de formateur-rice comporte des avantages variés qui dépendent fortement de la personnalité de l'individu, de la situation de travail et des pressions mutuelles ressenties. Certaines personnes apprécient d'être entourées de jeunes et mettent en avant le fait que chaque apprenti.e est différent.e et que cela ouvre de nouveaux défis. La plupart aiment observer le développement d'une jeune personne au cours de l'apprentissage et sont heureux de pouvoir y contribuer. Certaines personnes sont plus pragmatiques et conçoivent davantage la formation comme un nécessaire " retour sur investissement ». Après avoir investi leur temps et leur énergie à transmettre les compétences les plus fondamentales au novice, ils/elles souhaitent en récolter les fruits et tirer parti de leur main-d'œuvre (Bahl, 2018, pp. 273-281) ${ }^{8}$.

Nombre de personnes interrogées ont déclaré que le contact avec des jeunes et le fait de répondre à leurs besoins permettaient de rester jeunes et dynamiques. D'autres, en revanche, ont souffert de conflits avec les apprenti.e.s et considèrent que leur manque de discipline et leurs comportements représentent une énigme et un fardeau (Bahl \& Blötz, op. cit.). Sur un plan positif, les questions soulevées par la présence des apprenti.e.s obligent le/la formateur.rice à s'interroger et à mettre à jour en perma-

8. À terme, les apprenti.e.s déchargent leurs tuteur.rice.s de certaines tâches. 
nence ses connaissances, qu'il s'agisse des connaissances-métier ou des connaissances pédaggiques. En répondant aux questions des novices, les formateur·rice-s consolident en permanence leur statut d'expert et réaffirment leur statut d'employé compétent. Maurines souligne l'importance de cette dynamique pour les individus, mais aussi pour le développement des connaissances du personnel en tant que tel :

" [...] Le fait de former un collègue permet aussi au formateur de relativiser ce qu'il sait: il est obligé de sinterroger sur ses connaissances. Cette réactivité du professionnel face à son savoir est importante, puisque ce dernier est en perpétuelle constitution et transformation, les procédés de fabrication n'étant pas tous stabilisés. En bref, former quelqu'un, c'est prouver à soi et aux autres que l'on est compétent. " (op. cit., p. 11)

De même, plusieurs études ont révélé ce lien entre formation et innovation sur le lieu de travail (cf. Floro \& Raso, op. cit. , p. 92). Ces auteurs évoquent le "tutorat comme levier du développement». Ils soulignent qu'il se caractérise par une coopération mutuelle et un renversement de la relation de formation : "Dans le trio formateurl formélcollectif, si chacun des éléments est un chercheur, il est aussi médiateur de ses propres processus de développement. Chacun gagne en efficience en participant à la formation de ses pairs. " (Ibid., p. 93)

Si l'on peut tracer un parallèle entre cette dynamique et la formation en apprentissage, des différences cruciales subsistent entre les cas décrits, notamment quant au statut des personnes formées. Les apprenti.e.s du système en alternance souffrent d'un statut bien plus faible au sein de la hiérarchie de l'entreprise que celui des personnels classiques ou des nouvelles recrues qui rejoignent l'entreprise, car ils/elles n'ont pas encore acquis de qualification professionnelle. En outre, les entreprises de formation allemandes ne peuvent pas toujours promettre de garder les apprenti.e.s après l'obtention de leur examen final, ou n'ont même pas l'intention de le faire.

Comme nous l'avons évoqué plus haut, le lien entre les apprenti·e·s et les formateur·rice.s est crucial pour la transition vers le monde du travail et la réussite globale de l'apprentissage. Une relation perturbée est susceptible de menacer l'exécution du contrat de formation dans son ensemble (cf. Bahl, 2018, p. 274 sq. ; Masdonati \& Lamamra, 2009, p. 348s.). Une personne dotée de moins de compétences pédagogiques et moins apte à évaluer son propre comportement risque davantage de rejeter la faute sur l’apprenti.e (Bahl, 2012 ; Bahl \& Blötz, op. cit.).

Il peut donc être commode de répartir la responsabilité entre plusieurs employé.e.s, pratique courante dans les grandes entreprises et celles de taille moyenne, si les deux partenaires ne s'entendent pas bien. Toutefois, en général, les formateur·rice-s essaient d'éviter de telles situations. Notamment dans les secteurs où l'apprentissage représente une longue tradition, les formateur-rice-s s'identifient fortement à leur responsabilité morale vis-à-vis des jeunes et s'efforcent de protéger leurs apprenti-e.s le mieux possible en cas de difficultés dans l'entreprise ou à leur domicile. La citation suivante, 
recueillie dans le cadre du projet du BIBB, auprès d'un directeur de formation d'une grande entreprise de métallurgie, témoigne de cette posture protectrice :

"Les formateurs sont généralement le principal, voire le seul interlocuteur des apprentis. Ils acceptent également la responsabilité directe qui en résulte. Si les formateurs ne parviennent pas à apporter leur aide, les conséquences directes sont généralement négatives pour les apprentis, et les formateurs le ressentent également directement. Le résultat est une 'obligation d'agir' compulsive, même si les structures de l'entreprise ne le permettent pas, et les formateurs se retrouvent souvent à agir hors de leur champ d'activité et de responsabilité réel pour compenser les faiblesses de la structure de l'entreprise."

Outre à la dépendance des apprenti.e.s, cette citation fait également référence à la dépendance mutuelle entre les formateur-rice-s. et les apprenti-e.s. Le statut généralement faible des apprenti.e.s au sein du personnel, notamment des mineurs, induit une pression morale sur leurs supérieurs qui se sentent obligés de les aider par tous les moyens possibles à réussir leur formation, même si le soutien de l'entreprise est faible. Parallèlement à cela, la direction et le collectif de travail ont tendance à évaluer la performance du/de la formateur.rice à l'aune de la réussite de l'apprenti.e. Prenant le contrepied de toute considération pédagogique, l'évaluation des aptitudes et des compétences du/de la formateur.rice dans sa mission est généralement réalisée à l'aune de la progression de l'apprenti·e (cf. Besozzi, Perrenou \& Lamamra, op. cit., p. 60s.). Cette évaluation peut être néfaste si le niveau de l'apprenti.e est faible et qu'il/elle a de mauvais résultats.

\section{Les perturbations de la dynamique, dues au changement de la culture du travail}

Comme l'a montré l'analyse de la relation entre les trois principaux acteurs du mandat de formation dans ce modèle, l'équilibre entre eux est fragile. Sur le lieu de travail, de nombreuses circonstances sont susceptibles de menacer l'échange potentiellement bénéfique de reconnaissance sociale et professionnelle. Les études de cas ont révélé le très fort impact qu'exerce le contexte organisationnel sur la position et le rôle du/de la formateur.rice. Les formateur·rice-s désigné.e.s officiellement souffrent d'un handicap institutionnel dans la mesure où ce sont des personnels dépendants et que, dans le même temps, ils officient comme des employeurs face aux apprenti.e.s. Leur réussite personnelle en tant que formateur-rice dépend, d'une part, du soutien qu'apporte la direction à leur tâche et, d'autre part, de la motivation des jeunes. Outre l'organisation générale du travail dans l'entreprise et le secteur concernés, leur champ d'action est largement tributaire de leur position individuelle dans la hiérarchie de l'établissement. Si ces personnes sont ellesmêmes cadres intermédiaires ou bien s'entendent particulièrement bien avec leur supérieur hiérarchique, elles sont davantage susceptibles d'adapter et d'améliorer leurs activités 
de formation selon leurs besoins et ceux de leurs apprenti.e.s que quelqu'un au profil de poste plus restreint.

La motivation à s'impliquer dans l'apprentissage dépend d'une relation mutuelle et triangulaire entre formateur.rice.s, apprenti.e.s et direction. Les processus globaux de réorganisation, comme la sous-traitance/l'externalisation ou la segmentation, ont un impact sur cette configuration. La pression croissante sur les coûts et l'accélération des processus de travail ne font qu'exacerber les conflits intérieurs des formateur-rice-s liés au temps et aux objectifs. L'existence de telles difficultés a également été relevée dans d'autres contextes de formation (cf. Besozzi, Perrenoud \& Lamamra, op. cit. ; Boudet \& Triby, op. cit. ; Nativel, op. cit.).

Dans un contexte où le profit est moteur, consacrer des heures de travail à instruire des apprenti.e.s n'est désormais plus une évidence. Quel que soit le secteur, les formateur-rice-s de notre échantillon allemand n'ont eu de cesse de relater des problèmes de légitimité au sein des personnels. Sous la pression des exigences de rentabilité accrue, ils/elles sont tenu.e.s de prouver en termes économiques la légitimité de leur fonction de formateur.rice. Cette baisse de soutien de la direction fait ainsi peser toute la responsabilité de la formation sur les épaules des personnels.

Toutefois, la plupart des personnes qui ont répondu étaient peu préparées à faire quoi que ce soit pour s'opposer à cette tendance : certaines ont adopté une posture défensive, d'autres ont même intériorisé et repris à leur compte la fonction de formation comme un rôle exercé " à perte ", comme l'illustre cette déclaration d'un formateur à plein temps : "Parce que nous ne gagnons pas d'argent, dans ce sens, nous ne sommes pas productifs (rires étouffés), n'est-ce-pas?"

Pour l'étude du cas d'un groupe spécialisé en technologies de l'information et de la communication, coté en bourse, nous avons rencontré des formateur-rice-s dont les primes ont été réduites parce qu'ils/elles avaient investi des heures de travail dans la formation des apprenti.e.s plutôt que de faire de la facturation clients. L'un d'eux, tellement frustré après des discussions interminables avec différents supérieurs hiérarchiques qui lui demandaient tous de légitimer son intérêt " personnel » et d'établir les plans de formation, a fini par démissionner de cette fonction.

Ces exemples révèlent que les objectifs à court terme, les intérêts et le profit économique peuvent involontairement induire une externalisation de la formation vers un centre de formation indépendant ou même un abandon total de la formation par apprentissage. Le directeur de formation cité ci-dessus, qui se déclarait être " entre le marteau et l'enclume ", fait toutefois figure d'exception positive. En effet, face aux menaces exprimées par la direction d'externaliser la fonction de formation dans son ensemble, il a opté pour la stratégie offensive afin de se défendre, ainsi que son équipe. En mettant en avant le fait qu'ils "produisent quelque chose ici, plus exactement, des travailleurs qualifiés", ils ont adapté la formation au discours de la nouvelle philosophie d'entreprise de la direction et 
ont fini par réussir à asseoir la formation en apprentissage comme un "processus central » de leur entreprise (Bahl \& Blötz, op. cit.).

Si l'article de Maurines, daté des années 1990, n’évoque pas de restrictions de la formation dues à l'accélération des processus de travail, elle souligne toutefois un pré-requis important pour la garantie de son modèle industriel d'échange social : "En effet, le système d'échange ici décrit n'est pertinent qu'en référence à une culture d'atelier et non au niveau de l'entreprise. En effet, le hiérarchique ne peut prétendre rendre une contre-prestation dans un service où il n'a pas d'autorité. " (op. cit., p. 13)

Une " culture d'atelier ", fondée sur la confiance et la réciprocité, est un pré-requis pour la reconnaissance de l'apport du/de la formateur.rice. Elle dépend des relations personnelles et des possibilités dont disposent les supérieurs hiérarchiques pour soutenir les formateur-rice-s et façonner leur lieu de travail. Les réorganisations permanentes d'entreprises, la fusion avec d'autres entreprises bien plus grandes et souvent globales, ainsi que l'inflexion de l'orientation des différents départements vers des " centres de profit " ou encore l'externalisation de la formation effectuée alors au titre de service distinct sont autant d'éléments qui perturbent de plus en plus cette culture d'atelier.

\section{Conclusion}

La mise en regard des résultats de l'étude allemande sur l'apprentissage et des données empiriques tirées de plusieurs études de cas, menées dans le contexte français, fait émerger de nombreux parallèles, au-delà des contextes de travail et des objectifs de formation.

La majorité des formateur-rice-s et des tuteurs se consacrent à la formation au titre d'activité secondaire en plus de leur travail habituel. Ils le font non pas dans un cadre pédagogique, mais dans un contexte à dominante professionnelle et productiviste.

Comparé à la notion assez proche de "configuration tutorale », proposée par Pelé-Peycelon \& Veillard (op. cit.), le modèle industriel tripartite des partenaires du mandat de formation, formulé par Maurines (op. cit.) et développé dans le présent article, s'avère plus apte à révéler la dynamique sociale distincte des relations de travail. Afin de répondre aux défis modernes des cultures de travail en mutation, ce modèle devrait toutefois être redéfini, étendu à davantage de partenaires et alimenté par des recherches détaillées dans des secteurs choisis qui sont particulièrement touchés par la mondialisation.

Nous l'avons vu, mettre l'accent sur les formateur·rice·s, considérée.e.s comme des agents et des enseignants individuels, comme le font généralement les politiques européenne et allemande d'EFP visant leur professionnalisation, occulte les interdépendances particulières marquant la fonction de formateur.rice, ce·tte dernier.e étant par ailleurs un membre du personnel.

En outre, ces politiques présentent un biais pédagogique et ne reconnaissent pas suffisamment les défis auxquels sont confrontés les formateur-rice.s dans un cadre économique 
mais aussi dans des conditions de formation de plus en plus limitées. Kunégel a d'ores et déjà mis en garde, à juste titre, la France : "[...] Nous devons être vigilants contre les tentatives d'évaluer le tutorat à l'aune des pratiques pédagogiques à l'auvre dans le système scolaire. Les tentations sont encore fortes de transformer les tuteurs en pédagogues lors des stages de formation" (op. cit., p. 11). Ceci s'applique également au débat politique allemand.

Ainsi, plusieurs raisons plausibles expliquent que les deux certificats pédagogiques, promulgués en 2009 pour professionnaliser les formateur-rice.s en entreprise au-delà de leur qualification de base $(A E V O)$, celui de " pédagogue certifié de formation initiale et continue " ("Geprüfter Aus- und Weiterbildungspädagoge ") et celui de " pédagogue certifié de formation professionnelle " ("Geprüfter Berufspädagoge ") (Hensen \& HippachSchneider, op.cit., p. 14s.), ont un statut modeste dans le cadre de l'entreprise. L'industrie est généralement réticente à investir dans de telles certifications pour des motifs économiques et la direction comme les formateur.rice.s ne perçoivent pas l'avantage qu'apporte le contenu proposé (Bahl \& Blötz, op. cit.). Il conviendrait pourtant de promouvoir le concept d'apprentissage sur le lieu de travail en soulignant qu'il comprend bien plus que des situations de formation spécifique et qu'il requiert une stratégie complète de soutien émanant de l'organisation dans son ensemble.

Le développement professionnel des formateur-rice-s sur le lieu de travail nécessite initialement une réflexion systématique quant au rôle de chacun.e dans le contexte de l'entreprise. Ceci va dans le sens de l'appel lancé par Pelé-Peycelon \& Veillard à mener " une réflexion avec les tuteurs participants sur leurs propres réseaux de ressources, en interne de leur entreprise et aussi en externe" (op. cit., p. 81). Un renforcement de la prise de conscience et de l'estime de soi quant au rôle de facilitateur-rice d'apprentissage est un pré-requis essentiel au développement de stratégies personnelles pour négocier face aux exigences contradictoires auxquelles sont confrontés les formateur-rice.s.

Le présent article constitue une première et modeste tentative pour identifier les points communs et les différences concernant le statut de formateur.rice dans plusieurs contextes nationaux et sous divers régimes de formation. Des recherches ultérieures permettront de mobiliser une méthodologie comparative dès les prémices de la collecte de données empiriques. Ceci permettra d'extraire tout le potentiel d'un travail analytique profondément ancré dans les pratiques telles qu'elles sont vécues sur le lieu de travail et d'en tirer les enseignements afin d'améliorer le soutien aux formateur·rice.s.

\section{Bibliographie}

Bahl A. (2012), «Ausbildendes Personal in der betrieblichen Bildung: Empirische Befunde und strukturelle Fragen zur Kompetenzentwicklung », in Ulmer P. ; Weiß R., Zöller A. (Eds.), Berufliches Bildungspersonal - Forschungsfragen und Qualifizierungskonzepte, Bielefeld : W. Bertelsmann, pp. 21-43. 
Bahl A. (2018), Die professionelle Praxis der Ausbilder. Eine kulturanthropologische Analyse. Frankfurt/Main, New York : Campus.

Bahl A., Blötz U. (2012), Die Situation des ausbildenden Personals in der betrieblichen Bildung (SIAP), Forschungsprojekt 2.2.301, Laufzeit I-09 bis II-12, Abschlussbericht, Bonn : Bundesinstitut für Berufsbildung, 65 p. - URL : https:/www2.bibb.de/bibbtools/tools/dapro/data/documents/pdf/eb_22301.pdf

Bahl A., Brünner K. (2013), „40 Jahre Ausbilder-Eignungsverordnung - Eine Bestandsaufnahmezu ihrer pädagogischen Relevanz für betriebliches Ausbilderhandeln“, Zeitschrift für Berufs- und Wirtschaftspädagogik 109, 4, pp. 513-537

Barbier J. (1996), «Tutorat et fonction tutorale», Recherche \& Formation 22, pp. 7-19.

Besozzi R., Perrenoud D., Lamamra N. (2017), « Le temps au cœur des contraintes des formateurs et formatrices en entreprise ", Revue économique \& sociale, 1, pp. 53-68.

Billett S. (2002), "Workplace Pedagogic Practices: Co-Participation and Learning ", British Journal of Educational Studies, 50(4), pp. 457-481.

Blau P.M. (1964), Exchange and power in social life, New York: Wiley.

Boudet L., Triby E. (2016), «Le tutorat aux prises avec les contraintes du fonctionnement de l'organisation ", Éducation permanente, n 206 , 1, pp. 139-148.

The Bruges Communiqué on enhanced European Cooperation in Vocational Education and Training for the period 2011-2020 (2010), Communique of the European Ministers for Vocational Education and Training, the European Social Partners and the European Commission, meeting in Bruges on 7 December 2010 to review the strategic approach and priorities of the Copenhagen process for 2011-2020 - URL : https://www.eqavet.eu/ Eqavet2017/media/Documents/brugescom_en.pdf

Busse G. (2011), Der Ausbilder in der betrieblichen Ausbildung, Düsseldorf: Hans-BöcklerStiftung (Betriebs- und Dienstvereinbarungen / Kurzauswertungen) - URL : http://www. boeckler.de/pdf/mbf_bvd_ausbilder.pdf

Duc B., Perrenoud D., Lamamra N. (2018), «Les compétences transversales à partir du point de vue des formateurs et formatrices en entreprises : entre objets de formation et critères de sélection ", Éducation \& Socialisation, 47 - URL : http://journals.openedition.org/edso/2818; DOI : 10.4000/edso.2818

Filliettaz L. (2011), «Collective guidance at work: A resource for apprentices?», Journal of Vocational Education and Training, 63, 3, pp. 485-504.

Floro M., Raso M.-V. (2016), «Tutorat collectif et renversement de la logique de formation ", Éducation permanente, 206, 1, pp. 87-95.

Hensen K.A., Hippach-Schneider U. (2016), Supporting teachers and trainers for successful reforms and quality of vocational education and training: mapping their professional deve- 
lopment in the EU - Germany. Cedefop ReferNet thematic perspectives series - URL : http://libserver.cedefop.europa.eu/vetelib/2016/ReferNet_DE_TT.pdf

Kunégel P. (2012), Analyse des pratiques des maîtres d'apprentissage en situation de travail., Biennale internationale de l'éducation de la formation et des pratiques professionnelles. Paris - URL : https//halshs.archives-ouvertes.fr/halshs-00867025

Le Mouillour I., Geiben M. (2016), «L'engagement des entreprises dans le dispositif de formation professionnelle en Allemagne ", Revue internationale d'éducation de Sèvres, 71, pp 63-72.

Masdonati J., Lamamra N. (2009), "La relation entre apprenti-e et personne formatrice au cœur de la transmission des savoirs en formation professionnelle ", Revue suisse des sciences de l'éducation, 31, 2, pp. 335-353.

Maurines B. (1997), « Anthropologie de l'échange social, Apprentissages professionnels et dynamiques des relations au travail ", Formation Emploi, n 60, pp. 3-16.

Nägele C., Neuenschwander M. (2016), «Apprentice-trainer relationship and work group integration in the first months of an apprenticeship", Empirical Research in Vocational Education and Training 8, 4- URL : http://www.ervet-journal.com/content/8/1/4

Nativel J.-B. (2016), «Le tutorat à la Banque de France : vers un nouveau type d'apprentissage ", Éducation permanente, 206, 1, pp. 159-169.

Pätzold G., Drees G., Lietz M. (1986), «Haupt- und nebenberufliche Ausbildertätigkeit im Metallbereich, Aus den Ergebnissen des Forschungsprojekts,Ausbilder“», Berufsbildung in Wissenschaft und Praxis, 5, pp. 134-138.

Pelé-Peycelon M., Veillard L. (2016), « Des configurations comme resources pour devenir tuteur ", Recherche \& Formation, 83, 1, pp. 69-83.

Schlösser M., Drewes C., Osthues E. (1989), Vom Lehrgesellen zum Betriebspädagogen, Eine empirische Untersuchung zur Professionalisierung betrieblicher Ausbildertätigkeit, Frankfurt/Main, New York: Campus. 